\title{
CONSIDERAÇÕES SOBRE O TRATAMENTO CIRÚRGICO DO MELANOMA MALIGNO ANORRETAL
}

\author{
ASPECTS OF SURGICAL MANAGEMENT OF ANORECTAL \\ MALIGNANT MELANOMA
}

\author{
Marcelo Fernandes Rangel, TCBC-PB ${ }^{1}$ \\ Marcus Valério Maia da Silva, ${\mathrm{ACBC}-\mathrm{PB}^{2}}^{2}$ \\ Francisco de Sales Moreira Pinto, TCBC-PB ${ }^{2}$ \\ Manoel Jeovah Colaço Fernandes, TCBC-PB ${ }^{3}$ \\ Cássio Virgílio C. Oliveira, $\mathrm{ACBC}-\mathrm{PB}^{2}$ \\ João Batista Ribeiro Simões, TCBC-PB ${ }^{4}$
}

\begin{abstract}
RESUMO: São analisados os resultados do tratamento cirúrgico em cinco casos de melanoma maligno anorretal (MMAR). Um paciente encontrava-se no estádio clínico I, dois no estádio clínico II e dois no estádio III. Todos os pacientes foram operados, sendo quatro submetidos a excisão local do tumor, e um a amputação abdômino-perineal do reto. Este último sobreviveu por apenas sete meses, enquanto uma paciente, estádio clínico II e submetida apenas à excisão local, sobreviveu por 168 meses. Os autores questionam a validade do estadiamento clínico I ( tumores localizados ) como fator prognóstico, já que desconsidera o grau de penetração do tumor na parede anorretal. Sugerem ainda a realização de estudos multicêntricos, para que se possa estabelecer uma uniformidade terapêutica para o MMAR.
\end{abstract}

Unitermos: Neoplasia anorretal; Melanoma maligno; Tratamento cirúrgico; Sobrevida.

\section{INTRODUÇÃO}

Apesar da raridade do melanoma maligno anorretal (MMAR), que representa menos de $1 \%$ dos tumores malignos, esta é a terceira localização mais freqüente dos melanomas, seguindo-se à pele e olhos. ${ }^{1}$ Este aspecto adquire importância fundamental no que se refere ao diagnóstico nas fases iniciais da doença, considerando que o sucesso do tratamento cirúrgico, em termos de recidiva e sobrevida, depende mais do estádio da neoplasia do que da agressividade da terapêutica cirúrgica empregada. ${ }^{2,3}$ Além disso, na maioria dos casos, os sintomas podem simular afecções benignas, aspecto que condiciona a obrigatoriedade do estudo histopatológico em todos os casos de ressecções de mamilos hemorroidários, fístulas, plicomas, ou de outras lesões da região anorretal. ${ }^{4}$

Até o momento, não existe uniformidade, na literatura, quanto aos métodos de tratamento do MMAR. Da mesma forma, os resultados publicados são extremamente confli- tantes, com relatos de sobrevida média variável de 2,7 meses $^{5}$ até 30 meses $^{6}$ em pacientes submetidos a tratamento cirúrgico.

O objetivo da presente publicação é apresentar os resultados do tratamento cirúrgico em cinco casos de MMAR, enfatizando aspectos controversos, como a correlação entre estadiamento clínico, operação realizada, terapia adjuvante e sobrevida média dos pacientes.

\section{PACIENTES E MÉTODOS}

No período de outubro de 1974 a setembro de 1995 foram diagnosticados e tratados cinco casos de MMAR, no Hospital de Câncer Napoleão Laureano, em João Pessoa-PB.

Houve predomínio do sexo feminino, na proporção de 4:1, e os extremos de idade foram de 46 e 79 anos, com média de 60 anos. À exceção de um paciente de cor parda, todos os demais pertenciam à raça branca. Em relação ao quadro clínico, quatro pacientes perceberam tumoração na região anal.

1. Professor Adjunto do Departamento de Cirurgia do CCS-UFPB. Chefe do Serviço de Cirurgia Abdominal do Hospital de Câncer Napoleão Laureano.

2. Cirurgião do Serviço de Cirurgia Abdominal do Hospital de Câncer Napoleão Laureano.

3. Professor Adjunto do Departamento de Cirurgia do CCS-UFPB. Cirurgião do Hospital de Câncer Napoleão Laureano.

4. Professor Adjunto do Departamento de Cirurgia do CCS-UFPB. Diretor do Hospital do Hospital de Câncer Napoleão Laureano.

Recebido em 1/7/97

Aceito para publicação em 6/7/98

Trabalho realizado no Serviço de Cirurgia Abdominal do Hospital de Câncer Napoleão Laureano - João Pessoa -PB. 


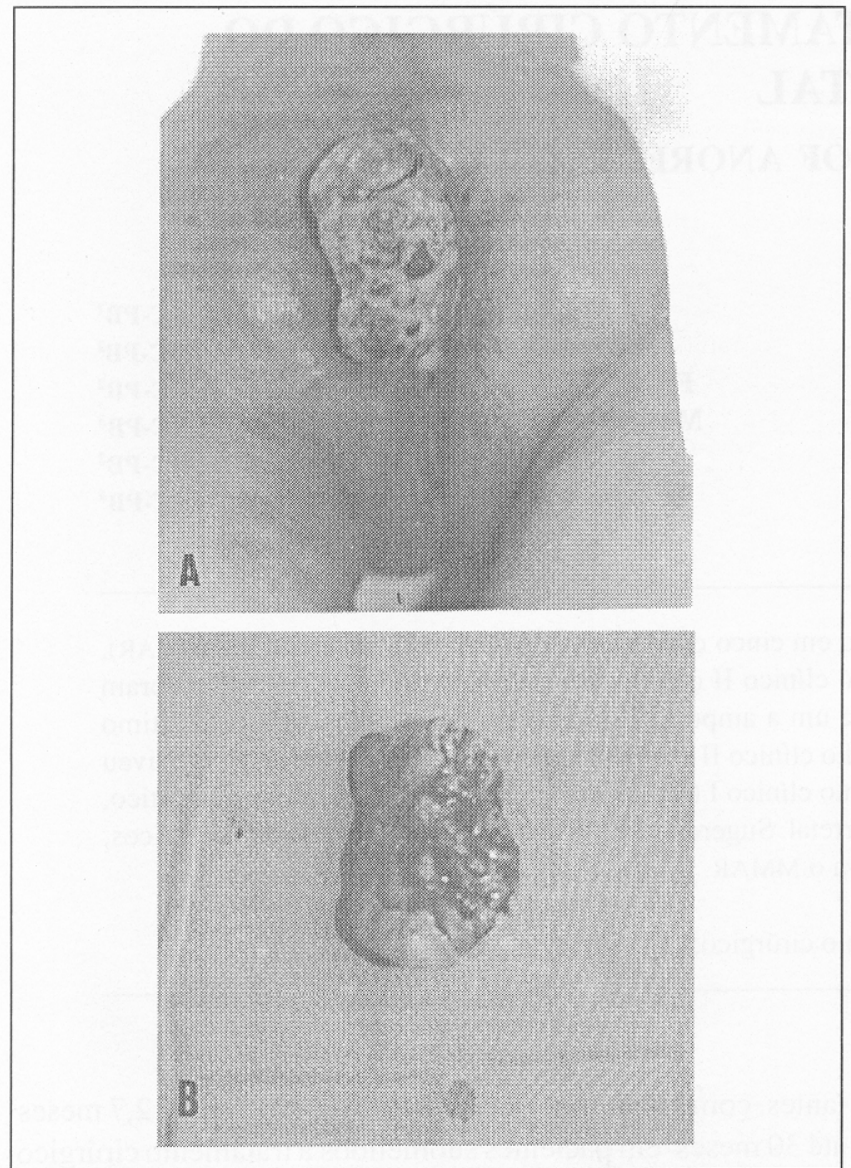

Figura 1-A) Melanoma maligno amelanótico do canal anal. B) Peça cirúrgica ressecada

Dois referiram sangramento retal, e os outros dois queixavamse de dor local. Obviamente, alguns pacientes apresentavam mais de uma queixa clínica. Quanto à forma de apresentação, quatro lesões eram polipóides, e uma delas, amelanótica (Figura 1). Em todos os casos, o diagnóstico foi realizado por meio de exame proctológico e confirmado por biópsia da lesão.

No presente estudo, utilizamos o estadiamento clínico (apud Slingluff et al), ${ }^{3}$ que classifica o paciente em três categorias: estádio I - tumores localizados; estádio II - comprometimento de linfonodos regionais (perirretais e/ou inguinais); e estádio III - metástases viscerais ou doença disseminada.

Em relação à modalidade terapêutica empregada, todos os pacientes foram operados. Em quatro, foi realizada excisão local do tumor (Figura 2), e um foi submetido a amputação abdômino-perineal do reto (AAPR). Em dois casos, foi instituída quimioterapia adjuvante, e um paciente foi submetido a radioterapia e imunoterapia inespecífica com BCG oral.

\section{RESULTADOS}

O tamanho do tumor variou de $4 \mathrm{~cm}$ a $8 \mathrm{~cm}$ (média $=5,6 \mathrm{~cm}$ ). Quanto ao estadiamento clínico (EC), um paciente apresentava-se no estádio I, dois no estádio II e dois no es-

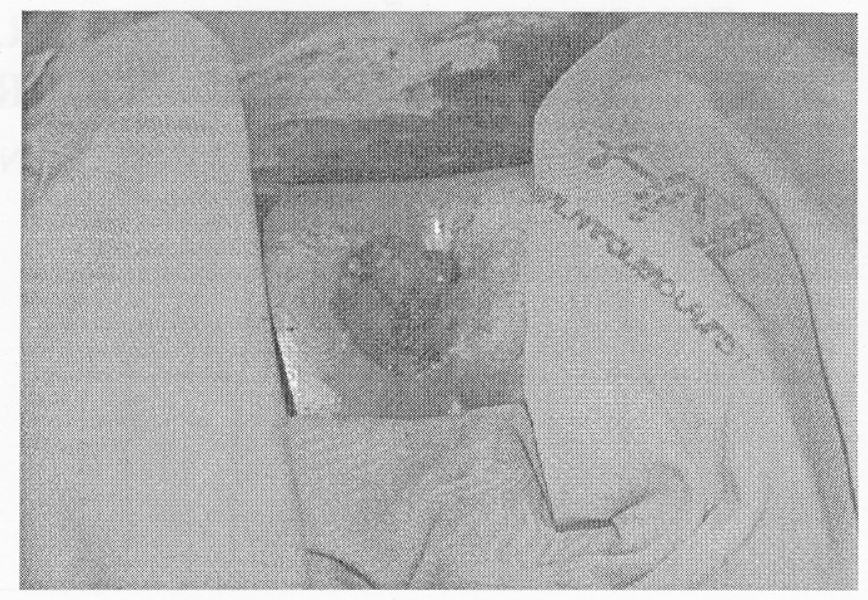

Figura 2 - Aspecto final da excisão local (mesmo caso da figura anterior). Ferida operatória aberta, para cicatrização em segundo tempo.

tádio III. Não houve complicacões ou mortalidade operatórias. O paciente submetido a AAPR já apresentava comprometimento de linfonodos inguinais, por ocasião do diagnóstico, e foi submetido a linfadenectomia inguinal trinta dias após a primeira intervenção cirúrgica. Como terapêutica adjuvante, recebeu radioterapia e imunoterapia inespecífica com BCG oral, porém sobreviveu por apenas sete meses.

Um paciente com doença disseminada, e outro, com metástases hepáticas, foram submetidos a quimioterapia adjuvante e sobreviveram por 8 e 17 meses, respectivamente. Uma paciente submetida a excisão local do tumor apresentou recidiva local 12 anos após a operação, sendo então reoperada (excisão local ampliada). Dois anos após a segunda intervenção, evoluiu com disseminação da doença, vindo a falecer. Surpreendentemente, esta paciente sobreviveu por 14 anos (168 meses), sem que tenha sido instituída nenhuma modalidade terapêutica adjuvante (Tabela 1 ).

\section{Tabela 1}

Distribuição dos cincos pacientes portadores de melanoma maligno anorretal

\begin{tabular}{c|c|c|c|c|r}
\hline Sexo & Idade & Cor & Estádio Clínico & Metástase & Sobrevida \\
\hline F & 63 & B & II & Inguinal & 168 meses \\
F & 46 & B & III & figado & 17 meses \\
F & 79 & B & I & - & 13 meses \\
F & 51 & P & III & Figado, ovários & 8 meses \\
& & & & $\begin{array}{c}\text { linfonodos, epiplon } \\
\text { inguinal }\end{array}$ & 7 meses \\
M & 61 & B & II & \multicolumn{3}{c}{} \\
\hline
\end{tabular}

\section{DISCUSSÃO}

Os melanomas malignos anorretais originam-se primariamente dos melanócitos da linha pectínea, onde ocorre o desenvolvimento de massas tumorais, geralmente maiores que 
$2 \mathrm{~cm}$ e quase sempre ulceradas por ocasião do diagnóstico. O tipo polipóide é a forma de apresentação mais freqüente, conforme ocorreu nesta série ( $80 \%$ dos casos), porém a lesão pode ser plana e/ou não pigmentada, caracterizando o melanoma amelanótico. ${ }^{2,5,6} \mathrm{~A}$ localização no reto é atribuída à progressão cranial da lesão, constituindo tecido anal ectópico e manifestando-se como melanomas retais. ${ }^{2}$

São extremamente raros na raça negra, e a distribuição por sexo é relatada como predominante em mulheres, na proporção de $2: 1{ }^{6}$ Em nossa série, esta proporção foi de 4:1, embora a casuística seja pouco representativa.

As manifestações clínicas mais comuns são sangramento retal, dor local e presença de massa tumoral, percebida pelo paciente. ${ }^{4} \mathrm{O}$ diagnóstico usualmente é tardio, pois as queixas clínicas são superponíveis às 'afecções anorretais comuns, ${ }^{7} \mathrm{e}$ as lesões podem apresentar-se sem pigmentação característica em até $40 \%$ dos casos, ${ }^{2}$ conforme ocorreu em um de nossos pacientes (Figura 1).

Deve-se, portanto, diante de lesões suspeitas, pesquisar rotineiramente a presença de adenopatias inguinais e/ou hepatomegalia, que estão presentes em $10 \%$ dos casos. ${ }^{8}$

Do ponto de vista histológico, o MMAR pode ser confundido com outras neoplasias desta região, como os linfomas, os sarcomas, o carcinoma indiferenciado e o hemangiopericitoma maligno. ${ }^{5}$ Nesta circunstância, torna-se imperiosa a utilização da imuno-histoquímica, que possibilita o diagnóstico diferencial através do antígeno melanótico específico (EMA), do antígeno leucocitário comum (CLA), e da proteína S-100. ${ }^{9}$ A classificação de Clark, universalmente aceita para o estadiamento histopatológico desses tumores, não pode ser empregada para os MMAR, devido à não correspondência das camadas cutâneas. ${ }^{8}$

Atualmente, utiliza-se o estadiamento clínico (EC), que classifica as lesões em: estádio I - tumores localizados; estádio II - comprometimento de linfonodos regionais (perirretais e/ ou inguinais ); e estádio III - presença de metástases viscerais ou doença disseminada. ${ }^{3} \mathrm{O}$ EC é bastante simples de ser utilizado, mas é passível de críticas, principalmente para os tumores classificados no estádio I, onde o estadiamento descrito desconsidera fatores importantes e seguramente relacionados ao prognóstico, como o grau de infiltração (profundidade) da lesão na parede anorretal. ${ }^{5}$
Do ponto de vista terapêutico, alguns autores afirmam que todos os métodos utilizados no tratamento do MMAR são válidos, no que concerne ao aumento da sobrevida. ${ }^{6}$ Entretanto, existe unanimidade, na literatura, quanto ao tratamento cirúrgico como método preferencial, embora persistam controvérsias sobre qual a melhor técnica a ser empregada: ressecção local ou AAPR. ${ }^{8}$

Os resultados publicados por Hickley e Magee ${ }^{6}$ reforçam o conceito da superioridade do tratamento cirúrgico, quando observaram uma sobrevida média de 17 meses após o diagnóstico, em pacientes não operados, e de 30 meses quando tratados cirurgicamente. Estes resultados são semelhantes aos obtidos no nosso serviço, onde verificamos uma sobrevida média de 42,6 meses em cinco casos operados, e contrastam de forma absoluta com os publicados por Vieira et al, ${ }^{5}$ onde a sobrevida média foi de apenas 2,7 meses para pacientes submetidos a AAPR.

No que se refere à correlação estadiamento clínico sobrevida, a observação de Vieira et $\mathrm{al}^{5}$ parece pertinente, no sentido de separar os pacientes EC I de acordo com os diferentes graus de penetração do tumor na parede anorretal.

Os nossos resultados corroboram esta observação, quando identificamos uma sobrevida de 13 meses para o único caso de diagnóstico no estádio I, semelhante à dos pacientes no estádio III (sobrevida média $=14$ meses ), e nitidamente inferior à dos pacientes no estádio II ( média de 87,5 meses ), conforme demonstrado na tabela 1 .

Apesar de Slingluff et al, ${ }^{3}$ em análise multivariada de prováveis fatores determinantes da sobrevida (estádio, sexo, idade, raça e forma de tratamento), terem observado significância estatística apenas na variável estádio da doença, acreditamos que o estadiamento clínico I (tumores localizados) é por demais simplista e deva ser modificado, objetivando um melhor planejamento terapêutico.

Analisando os relatos da literatura especializada, podese verificar que os métodos de tratamento do MMAR continuam individualizados, e até mesmo aleatórios, mesmo nos centros de referência.

Assim, considerando a raridade do MMAR, e os resultados controversos publicados na literatura, talvez a realização de estudos multicêntricos possa estabelecer uma uniformidade terapêutica para o melanoma maligno anorretal.

\begin{abstract}
In spite of being rare, the anorectal region is the third most frequent localization of the melanoma, following the skin and eyes. The results of treatment are conflictant, with variable averange survival of 2,7 months until 30 months in patients submitted to surgical treatment. In this study, the results of surgical treatment in five cases of malignant anorectal melanoma are presented. One patient was in clinical staging I, two in II and two in III. All of them have been operated, four submitted to local excision of the tumor and one to abdominoperineal amputation of the retum. Two patients were submitted to adjuvant chemiotherapy and one to radiotherapy and inespecific imunotherapy with oral BCG. The unique patients in the stage I had a survival of 13 months, similar to the patients III ( average survival = 14 months), and inferior to the patients $I I$ ( average survival $=87,5$ months $)$. The authors question the validity of the clinical stage I(localizated tumours) like a
\end{abstract}


prognostic factor, because it desconsider the penetration grade of the tumour in tha anorectal wall. The still suggest the realization of multicentric studies to allow the establishment of a therapeutic uniformity to the malignant anorectal melanoma.

Key Words: Anorectal neoplasm; Malignant melanoma; Surgical treatment; Survival.

\title{
REFERÊNCIAS
}

1. Quinn D, Selah C - Malignant melanoma of the anus in a nigro. Dis Colon Rectum 1977; 20: 627 - 631.

2. Campos FG, Habr-Gama A, Silva JH, et al - Melanoma maligno da região anorretal: apresentação de um caso e revisão da literatura. $A B C D$ 1990;4:102-124.

3. Slingluff CL, Volmer RT, Seigler HF - Anorectal melanoma: clinical characteristics and results of surgical managment in twenty-four patients. Surgery 1990;107:1-9.

4. Chiu SH, Unni KK, Beart RW - Malignant melanoma of anorectum. Dis Colon Rectum 1980;23:122-124.

5. Vieira RAC, Nakagawa WT, Rossi BM, et al - Melanoma maligno do canal anal- existe indicação para a amputação abdominoperineal? Acta Oncol Bras 1996;16:186-89.

6. Hickley EB, Magee D - Malignant melanoma of the anal canal. Irish Med J 1982;75:83 - 86.

7. Wanebo HJ, Woodruff JM, Farr GM, et al - Anorectal melanoma. Cancer 1981;47:89 -90.
8. Habr-Gama A, Souza Jr AHS - Câncer no ânus. In : Tratado de Clínica Cirúrgica do Aparelho Digestivo. Pinotti HW (Ed). Editora Atheneu, v. 2, São Paulo-SP, 1994, pp. 1.293-1.300.

9. Clemmensen OJ, Fenger C - Melanocytes of the anal canal epitelium. Histopatology 1991;18:1.237-1.241.

ENDEREÇO PARA CORRESPONDÊNCIA

Dr. Marcelo Fernandes Rangel

Rua Gal. Aldenor Quinderé, 112

58033-500 - João Pessoa - PB

\section{Congresso Brasileiro de Cirurgia}

\section{4 a 8 de julho de 1999}

\author{
RioCentro \\ Rio de Janeiro
}

